\title{
El estudio del lenguaje de labibliotecología y disciplinas afines
}

\author{
RAMIRO LAFUENTE LÓPEZ \\ Centro Universitario de Investigaciones Bibliotecológicas \\ de la UNAM, 04510, México D.F., Tel: (525)623-03-29 \\ E-Mail: lafuente@servidor.unam.mx
}
Trabajo recibido el
4 de octubre de 2000 * Trabajo aceptado el 10 de noviembre de 2000

\begin{abstract}
RESUMEN
Existen disciplinas en las que la actividad profesional genera conocimientos empíricos y utiliza un lenguaje muy ambiguo para expresarlos, lenguaje que además predomina en los ámbitos profesionales. Esto dificulta la difusión del conocimiento científico creado en los ámbitos docentes y de investigación dada la falta de instrumentos para facilitar la difusión del conocimiento que sirvan como referentes para la comunicación entre los ámbitos docentes y de investigación y los profesionales. A esto se añade el escaso interés en los estudios acerca de los corpus de términos de la disciplina, por ello en este artículo se abordan una serie de cuestiones acerca del análisis de términos, su forma de clasificarlos y su utilidad como métodos para construir sistemas de clasificación que sirvan como medio de comunicación.
\end{abstract}

PalabresClave Temindogáa đasificacón Lenguajeespecializadb

THE STUDY OF THE LANGUAGE OF LIBRARY SCIENCE AND CLOSE DISCIPLINES RAMIRO LAFUENTE-LÓPEZ

\begin{abstract}
There are those disciplines in which professional activity generates empirical knowledge whose diffusion is seriously encumbered by the oblique, ambiguous language of their research and teaching spheres. This situation is exacerbated by the lack of facilitating instruments, designed for the accurate and efficient dissemination of knowledge, which could serve as communication signposts between the teaching, research and professional realms of the discipline in question. Furthermore, because of a dearth of interest to study the body terms of a given discipline, this paper approaches a series of questions regarding the analysis of terms, their classification and their usefulness as tools for the construction of classification systems that serve as communication media.
\end{abstract}

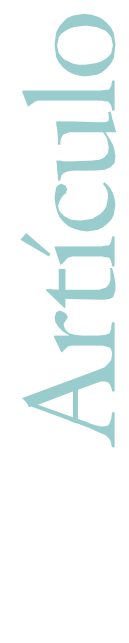

KeyWbrds Temindogy, Cassifiction Spedalized Language 


\section{INTRODUCCIÓN}

$\mathbf{E}^{1}$ estudio del lenguaje de la bibliotecología y disciplinas afines, entre otras finalidades, puede orientarse a comprender su papel como instrumento de comunicación del conocimiento. Una primera aproximación al lenguaje de las disciplinas antes mencionadas muestra que éste no está exento de contenidos ideológicos y culturales que se manifiestan en la diversidad de significados atribuibles a un mismo término. Esto induce, en una primera instancia, a considerar la imposibilidad de lograr una concepción unificada del conocimiento que se produce en el espacio de estudio de estas disciplinas. No obstante estudiar el lenguaje de estas disciplinas nos puede proporcionar elementos para conseguir que lo que ahora aparece como algo diferente o separado forme una organización y responda a finalidades comunes.

Un factor a considerar en los estudios del lenguaje de la bibliotecología y disciplinas afines, es la identidad de la comunidad que genera y utiliza este lenguaje; es decir, el conjunto de características que hacen de esta comunidad algo distinta de las otras, y que por tanto permite identificarla y delimitarla. La comunidad que utiliza el lenguaje especializado de estas disciplinas tiene pocos rasgos de cohesión en cuanto a las creencias acerca de los fenómenos objeto de estudio, y por tanto difícilmente puede generar reglas comunes para el uso del lenguaje, de manera que la identidad de la comunidad se refleja en una diversidad de modos de uso del lenguaje. En el caso de disciplinas como las que nos ocupan, en donde la actividad profesional genera conocimientos empíricos y utiliza un lenguaje muy ambiguo para expresarlos lenguaje que además predomina en los ámbitos profesionales, se dificulta la difusión del conocimiento científico generado en los ámbitos docentes y de investigación dada la falta de reglas para la comunicación del conocimiento. No existen, por ejemplo, manuales de estilo de publicación que sirvan como referentes para la comunicación del conocimiento.

El lenguaje especializado de una disciplina o área del conocimiento es una totalidad compleja que está hecha de entidades diferentes organizadas en distintos grados y jerarquías de subordinaciones y relaciones. En esta totalidad compleja aparece siempre un momento dominante y predominante que determina la estructura de todo el lenguaje y la finalidad de cada una de sus partes. Esto implica que existen periodos en los cuales las creencias dominantes de los sujetos que forman una comunidad epistémica dotan al lenguaje de una coherencia significativa. 
La interpretación y producción de términos ${ }^{1}$ en el ámbito de una disciplina o área del conocimiento supone procesos mentales relativos a la comprensión, la formulación, y el uso del conocimiento así como de otras estrategias de la dimensión cognitiva del discurso, como la oración y la palabra o conjunto de palabras que se utilizan para expresar un concepto o para describir hechos o eventos. Usualmente un solo significante, es decir, una palabra, frase, expresión o signo de una idea o de un pensamiento, o de una cosa material, ya sea un fonema o secuencia de fonemas o letras, constituye un signo lingüístico que transmite contenidos temáticos diferentes relacionados entre sí; por tanto, lo que se llama lengrajees, la mayoría de las veces, un conjunto de términos cuyo contenido es un discurso en varios niveles.

Los estudios sobre lenguaje también consideran las finalidades, metas y objetivos que se desean cumplir con éste, así como las características morfológicas de los términos que constituyen el compusdetéminosde un disciplina. El lenguaje de una disciplina puede estudiarse a partir del análisis de cada uno de los términos que lo componen de manera autónoma y lo convierten en medio para producir discursos significativos. Es el caso por ejemplo del estudio timlógicoomofdóġøade cada uno de los términos que integran un lenguaje especializado. Aunque también pueden estudiarse cada uno de los términos que componen el lenguaje, unos en relación con otros y con el contexto social en que se producen y aplican, y enfatizar los aspectos sociales del uso del lenguaje como medio de comunicación del conocimiento. En ambos casos se producen distintos tipos de conocimiento acerca del lenguaje.

En el caso de las disciplinas que nos ocupan es fundamental destacar que los fenómenos que estudia se nombran con términos que forman parte del lenguaje común y corriente, y que la mayoría de las veces se usan también estos términos para denotar conceptos derivados de la actividad científica, por lo que el estudio del lenguaje de estas disciplinas debe distinguir, identificar y delimitar esta diversidad de términos y significados, y organizarlos por medio de una nomenclatura que conduzca a la creación de un conjunto de principios y reglas que le permitan alcanzar una denominación inequívoca, única y distintiva de los taxones de los conceptos y objetos.

En una circunstancia dada algunos términos proyectan una diversidad de significados dependiendo de los distintos contextos culturales en los que se generan, situación que puede atribuirse a la dinámica social de las comunidades en las que se desenvuelve el uso y desarrollo del lenguaje. A veces esto deriva de la intencionalidad de las personas y se manifiesta en el manejo del lenguaje bajo aspectos ideológicos, o bien, en el uso de modismos de origen nacional. En otras ocasiones los significados del lenguaje están determinados por el avance intelectual, las creencias y los prejuicios que posee cada persona, o una comunidad en particular.

1 Término lo utilizamos en el sentido de la noción que alude a una palabra, un signo o conjunto de signos articulados que expresan una idea. 
Para entender los contenidos que representan los términos de que se compone un lenguaje especializado se necesita interpretar; es decir, para que se dé la comprensión del significado de un término no es suficiente con que haya información, debe también existir información en la memoria del sujeto que interpreta, que le permita interpretar. En este sentido son necesarios esquemas de conocimiento en la memoria del sujeto, que puedan ayudarle a hacer inferencias. Así, podríamos decir que la interpretación del significado de los términos de que se compone un lenguaje, depende del conocimiento apriai del sujeto que interpreta. Este conocimiento aprion, es un conjunto de conceptos relacionados entre sí, que le permiten al sujeto hacer las inferencias necesarias para significar.

La comunicación por medio del lenguaje especializado requiere de códigos que son conocidos apriorísticamente tanto por el receptor como por el emisor, y que en consecuencia constituyen todo aquello que restringe una elección arbitraria de los términos y componentes del lenguaje a utilizar. Podemos identificar a esto como el conjunto de reglas que establecen una previsibilidad más o menos acentuada en la comunicación, lo cual contribuye a poner orden en la combinación de los términos y sus significados, todo lo cual favorece la inteligibilidad ${ }^{2}$. En particular forman parte de estas reglas que establecen códigos de comunicación, las reglas de la gramática, de la lógica, de la sintaxis, las formas de validación del contenido, las formas aceptables de publicación, los prejuicios profundamente arraigados, etcétera, que sucesivamente constriñen la elección, el uso y el significado de las palabras.

Una de las reglas más elementales para organizar la comunicación en el ámbito de una disciplina es la construcción de un repertorio que represente una organización primaria del lenguaje, como es el caso de los diccionarios o vocabularios, concebidos como la simple enumeración de palabras admitidas como utilizables para comunicarse.

Sin comprender el significado de los términos que componen un lenguaje especializado y las reglas que rigen la organización de la comunicación por medio del lenguaje, no hay manera de interpretar los contenidos temáticos que tales términos expresan. La interpretación se basa en la interacción simultánea de los contenidos que nombra un término, así como en las reglas que rigen la organización de la comunicación, y los conocimientos del sujeto que interpreta. Sin embargo para comprender los significados de los términos de un lenguaje es necesario tener un sólido fundamento, un modelo abierto a cualquier desarrollo ulterior de la comprensión; es necesario pasar de la conciencia empírica a un conocimiento científico o reflexivo, despojándose de todo prejuicio teórico. El punto de partida es tratar de entender y definir, hacerse preguntas que finalmente conduzcan a establecer las formas que se utilizan para integrar y manejar un lenguaje especializado. Se trata, pues, de buscar

2 Sobre los aspectos que influyen en la comunicación escrita véase el capítulo 3: la Transmisión del mensaje cultural de la Obra de Abraham A. Moles.- SociodinamicadelaCultura- Buenos Aires, Paidos, 1978.- 334 p. 
respuesta para el problema de la validez de nuestro conocimiento sobre el lenguaje especializado de la bibliotecología y las disciplinas afines.

El sistema de comprensión de los contenidos temáticos de cualquier texto pasa por la comprensión de los términos que integran un lenguaje especializado, sobre todo el lenguaje especializado escrito, en tanto que ésta instaura un medio para percibir o expresar tanto lo que otros dicen como lo que nosotros queremos decir, y de dejarlo registrado en forma permanente. Todo tratamiento del lenguaje especializado necesariamente pasa por el estudio de la forma y el contenido de significados a que componen cada término. Así, decimos que el análisis de la lengua escrita implica a la forma y al contenido, donde en la primera se percibe para recuperar información, y el segundo se percibe e interpreta para la significación.

El estudio del lenguaje de una disciplina conlleva implícito el abordaje de los principios, métodos y fines sobre las formas de clasificar este lenguaje, con la intención de ordenar, jerarquizar y sistematizar los términos de que se compone. Su objetivo inmediato es contribuir a organizar las reglas de comunicación en el ámbito de una disciplina y lograr esquemas de clasificación que faciliten la organización y búsqueda de esos significados. No obstante este tipo de estudios contribuye también al desarrollo de la investigación científica acerca del uso y significado de los términos en diversas áreas temáticas, con el objeto de ampliar la capacidad de comunicación y de integrar los resultados de investigación sobre estos asuntos.

La clasificación de los términos que forman parte del lenguaje especializado de una disciplina o área del conocimiento, con base en la representación de la organización lógica del conocimiento en esa disciplina y en concordancia con una estructura jerarquizada que sea significativa para los sujetos que la utilicen, sería válida como método de análisis para determinar la significación de los términos de un lenguaje especializado. Empero esto crearía una estructura de clasificación que si bien mostraría el encadenamiento de términos a partir de la relación que existe entre las ideas que éstos expresan, no siempre resulta ágil y de fácil consulta para propósitos prácticos de búsqueda de términos y sus significados, aun cuando indudablemente puede tener un inapreciable valor como instrumento de investigación y de enseñanza-aprendizaje del lenguaje especializado.

\section{ORGANIZAR EL LENGUAJE DE UNA DISCIPLINA}

El estudio del lenguaje utilizado en la literatura hispanoamericana sobre bibliotecología, y ciencias de la información y documentación, puede darse a partir de las diversas formas de organizar los términos en torno a categorías representativas del conocimiento producido por estas disciplinas. En la literatura hispanoamericana, los términos que representan los contenidos temáticos tienen diversos valores de significación, un espíritu simplista buscaría eliminar dificultades y tendería a darle un "var la̛" unívoco a cada término, con la intención de evitar las confusiones que conlleva 
la significación, todo por querer "validar" los conceptos que representa cada término y de obtener así el significado propio de cada uno de ellos.

Sin embargo, en un lenguaje especializado los significados no derivan únicamente del significado propio de cada término, se desprenden también de las relaciones entre ellos y del orden en que se presenta esa relación. Cuando preguntamos sobre el significado de un término, lo que estamos haciendo es indagar sobre las relaciones que éste establece.

El universo que se puede determinar para clasificar los términos que integran el lenguaje de una disciplina puede definirse en tanto la bibliotecología, la documentación y las ciencias de la información se piensen como disciplinas, y los contenidos temáticos de su literatura expresen diversos conceptos susceptibles de relacionarse de diversas formas. La relación de contenidos temáticos se efectúa en razón de los significados propios de cada término y las finalidades que se persiguen al establecer la relación; es decir, la idea de vincular los contenidos se sustenta en la relación lógica que se desea establecer entre el significado o significados de un término y los propios de otro, con objeto de obtener un instrumento que haga posible conocer de manera sistemática el significado y las relaciones de los términos constitutivos de un lenguaje especializado.

Las relaciones que se establecen para vincular el significado de un término con el de otro responden a una sola estructura lógica de orden. Conforme se van desarrollando relaciones en un espacio de clasificación para vincular cada uno de los contenidos temáticos presentes en la literatura de una disciplina, se utiliza la heterogeneidad que sirve para delimitar cada uno de los contenidos temáticos, diferenciando cada uno de los términos entre sí. Pero a la vez la clasificación se sustenta en un sistema clasificador homogéneo que sirve para integrar un orden adecuado y para identificar y relacionar cada una de las partes que se están agrupando bajo un solo esquema. Así la ordenación de los términos de un lenguaje especializado obedece al propósito de ordenar las relaciones entre los distintos significados que remiten al término; de esta manera la clasificación nos permite establecer el tipo de relación que existe entre los diversos términos que componen un lenguaje.

El proceso de análisis para construir un esquema de clasificación que permita agrupar los términos pertenecientes a una disciplina, implica la aplicación de inferencias que permitan validar el significado que se le atribuye a esos términos. La intención es facilitar el manejo sistemático de los diversos significados que pueden atribuírsele a un término en particular, y de esta manera poder desprender las relaciones entre tales significados. En el caso de la literatura hispanoamericana sobre bibliotecología, ciencias de la información y documentación, es indispensable considerar las diversas formas en que se maneja el significado de los términos, dado que en ocasiones se presenta gran diversidad de significados debido a los contextos culturales en los que se producen, y a las condiciones mismas del desarrollo de las creencias y conocimientos de las comunidades de sujetos que aplican y manejan estas disciplinas. 
Un buen camino para abordar el estudio del lenguaje de una disciplina es reconocer las ideas y conceptos para luego buscar los verbos que mejor los representan, a fin de conocer no sólo la estructura morfológica de las palabras que integran el lenguaje sino también las acciones que éstas involucran.

\section{IMAGINAR UN LENGUAJE ES IMAGINAR UNA FORMA DE VIDA ${ }^{3}$}

Como el contenido de un lenguaje especializado está sujeto a una constante transformación empírica, las personas que usan y abusan del lenguaje de una disciplina van determinando tanto su construcción como su estabilización, en tanto que establecen un consenso acerca de un núcleo de términos y de significados de uso común. No obstante, esta misma estabilización del lenguaje crea también la necesidad de generar distintas formas de su uso, formas que están en relación con las maneras de jerarquizar y relacionar las palabras con objeto de expresar nuevas ideas o de designar nuevos fenómenos o hechos. Wittgenstein introdujo la idea de juegosddlengrajecomo modo de para distinguir la diversidad de sus formas de uso. ${ }^{4}$

$\mathrm{Si}$ adoptamos la idea de juegos del lenguaje de Wittgenstein podríamos ejemplificar una diversidad de estos juegos en el ámbito de la bibliotecología y disciplinas afines; por ejemplo:

* Construir y probar hipótesis y teorías

* Describir la forma y contenido de los documentos

* Reportar algún evento

* Describir técnicas y procesos

* Especular acerca de un evento

* Describir servicios de información

* Describir sistemas de clasificación

* Realizar informes de actividades bibliotecarias

* Reportar resultados de búsquedas de información bibliográfica

Los juegos del lenguaje no son detectables examinando meramente la forma de las palabras, puesto que en este sentido todas ellas están uniformadas por las reglas de la gramática. Un juego de lenguaje implica ser capaz de jugarlo; es decir conocer las actividades asociadas con él y poder, por lo menos en principio, tomar parte en ellas. En otros términos, podríamos decir que existe una práctica asociada a cada una de las formas de uso del lenguaje, lo cual nos lleva a considerar que la comprensión importante del lenguaje es la que procede del lenguaje aplicadb, es decir, de las expresiones conectadas con actividades concretas.

3 Idea retomada de Wittgenstein.

4 Sobre esta noción de juegos del lenguaje. Cfr. Tomasini Bassols, Alejandro.- Lenguaje y Anti-meafísica: CaviladionesWinttgensteinianas- México: INBA/Interlínea, 1994.- 282 p. -- p. 31 y ss. 
Los significados del lenguaje ordinario suelen ser oscuros y confusos. Muchos términos son usados sin precisión, sus fronteras de aplicación son vagas, y a menudo una misma palabra puede tener varios significados y expresar conceptos diferentes. Esto sucede en el caso de conceptos vagos o imprecisos que expresan distintos tipos de significados en distintos niveles, como pudiera ser el caso del término Biblio tea que tiene diversos significados tanto en el lenguaje común como en el lenguaje teórico relativo a la bibliotecología y disciplinas afines. Al análisis reflexivo del lenguaje especializado le corresponde clarificar, distinguir y sistematizar cada uno de esos términos hasta llegar a un sistema ordenado de conceptos definidos con cierta precisión. Aunque se parta de los usos comunes del lenguaje, el análisis conceptual debe proponer una organización de las reglas de comunicación, que promueva nuevas formas de apreciación del uso del lenguaje.

Podemos llamar conceptos epistémicos al conjunto de nociones que se refieren a un determinado tipo de conocimiento y sirven para describirlo y difundirlo. Podríamos pensar que el análisis, la clarificación y la sistematización de los conceptos epistémicos parte del examen sobre los contenidos temáticos de un ámbito determinado del conocimiento. Sin embargo la precisión y claridad en el análisis de los términos epistémicos depende de las relaciones que éstos guarden entre sí como parte de un sistema de conceptos. El análisis de los significados con la finalidad de establecer esquemas de clasificación que los agrupen y ordenen de alguna manera, presupone la aceptación de la idea de que el conocimiento se produce y organiza como un sistema que se establece a partir de relaciones regidas por las reglas de la lógica.

El establecimiento de reglas lógicas que expresen las relaciones entre varios conceptos permite determinar con mayor precisión su pertenencia a determinada familia epistémica. Con todo, la formalización tiene que partir de un supuesto semántico:

las definiciones de los conceptos son previas a la formalización de las relaciones de significados entre diversos términos y no pueden provenir de ella; es decir no podemos desprender las definiciones y significados de los términos a partir de las relaciones entre éstos

Son esas relaciones, ya establecidas previamente por medio de la construcción de conceptos y las inferencias que se implican en toda definición conceptual, las que se formalizan. Por tanto el examen de los contenidos semánticos debe preceder a cualquier formalización de las relaciones que puedan establecerse entre los términos que conforman el corpus de un lenguaje especializado. Y ese examen no puede aludir solamente a un análisis formal de las palabras, debe incluir también las formas de uso de los conceptos en el lenguaje que está utilizando una comunidad, y tal análisis debe ser de carácter explicativo y abandonar cualquier intención de normalizar las formas de uso del lenguaje, para evitar reducir su examen a un simple estudio morfológico disfrazado de análisis semántico.

El análisis semántico de los términos que componen el lenguaje especializado de la bibliotecología y disciplinas afines debe pretender, ante todo, clarificar sus conceptos. Para ello tiene que partir de los significados usuales de los términos, examinar sus 
distintos usos en diferentes situaciones, y variarlas en ejemplificaciones sucesivas hasta dar con un núdeode significado que permanezca a través de todas las variantes. Ese núcleo puede expresarse en las condiciones necesarias y suficientes de aplicación del concepto. Clarificar el concepto es también determinar las notas esenciales del objeto al que se refiere; esto es, las notas invariables que se perciben al través del análisis de las variaciones de significado que se le atribuyen, y que debemos admitir como las peculiaridades que presenta un objeto que se representa por medio de un término en particular. Este proceso no difiere substancialmente del mútodbdelasvar niadones de la fenomenología. En efecto, se trata de tomar el significado como hilo conductara través de las variaciones de los ejemplares que lo cumplen, hasta quedarnos con un núdøinvariableque sólo desaparecería al desaparecer el significado. La clarificación conduce, a la vez, a precisar el concepto y a determinar las notas esenciales del objeto.

El análisis semántico no sólo le exige claridad a los conceptos, sino también distinción. Por ello delimitar el significado de un concepto respecto de otros representa la fase primaria de la clarificación conceptual, que además es la que permite percibir las primeras posibilidades de relación entre los términos que representan estos conceptos. El uso de varios términos en el lenguaje especializado para referirse a un mismo concepto, responde a menudo a distinciones reales en los objetos; las cuales son a veces de gran importancia teórica. Si tenemos dos términos que responden a sendos conceptos, y uno de ellos basta para describir adecuadamente determinados hechos, si queremos precisar la distinción entre ellos podemos preguntar en cada caso ¿cuál es la situación exacta que obliga a utilizar los dos conceptos?

Son necesarios nuevos caminos y formas para explicar, representar y organizar los contenidos y significados de los términos. Uno de los aspectos indicativos de las deficiencias del lenguaje de la bibliotecología y disciplinas afines es, por ejemplo, su falta de habilidad para comprender, explicar y representar por medio de términos unívocos, la naturaleza, dinámica, y evolución de algunos fenómenos que tienen una historicidad muy amplia, como el término biblictecaodbumento u otros relativamente nuevos y producto del desarrollo tecnológico como es el caso, entre otros, del dour mentodigtal. Aquí tenemos una circunstancia inversa a la mencionada en el párrafo anterior, ${ }^{5}$ en donde se tiene un solo término para representar fenómenos que pueden explicarse desde distintas perspectivas y que podrían dar lugar a distintos términos que se refieren al mismo fenómeno.

Una tendencia en la formulación de conceptos y términos que representen fenómenos complejos con un alto grado de historicidad ha sido la construcción de términos compuestos cuyo significado pretende tener cierto grado de generalidad, como es el caso del término: unidadesdeinfomadón por medio del cual se pretende aglutinar el significado de varios términos: biblictecaensusdivesasacepiones, cetrodeinformadón, 
centrodedoumetación Sin embargo este tipo de formulaciones crea más confusiones de las que despeja, puesto que no aglutina significados, únicamente crea un término que pretendidamente agrupa a otros. En este sentido el término biblioteca no sólo representa conceptos teóricos sino que también se utiliza para describir actividades. ${ }^{6}$

La funcionalidad y procesos de captura y conversión de los documentos digitales convierte en volátil la forma del documento. Muchos documentos digitales tienden a estar en constante transformación, como es el caso del la web. Es necesario desarrollar relaciones taxonómicas que faciliten el acceso a los contenidos temáticos de este tipo de documentos, en los que sus contenidos son parte de un proceso de evolución contante conforme pasa el tiempo. La práctica de fijar los contenidos temáticos de los documentos derivada del uso de la cultura sustentada en la imprenta no es necesariamente útil para comprender y explicar el fenómeno social que envuelve la creación y el uso de los documentos digitales.

¿Cómo podemos empezar a comprender las propiedades de los documentos digitales? Podríamos empezar por nombrarlos y describirlos con acciones verbales más que con sustantivos y adjetivos, puesto que la terminología sustentada en sustantivos se enfoca únicamente al tipo del documento y lo aísla del contexto social en que se produce y utiliza. Por ejemplo, CD-ROMnombra una tecnología que no denota las relaciones sociales, las prácticas ni las formas de organización del trabajo que implica cuando se utiliza como instrumento para registrar diversos tipos de documentos con finalidades tan diversas como archivar los documentos contables de una empresa o difundir los resultados de una investigación.

Los documentos digitales permiten o inducen la utilización colectiva de sus contenidos temáticos, y no porque esto no haya sido una práctica usual en la historia humana, sino porque la facilita, y además se realiza a través de prácticas derivadas de sistemas que predeterminan las formas de uso. Lo cual hace pensar en quién es realmente el que crea un documento digital y en cuál es su significado.

Las relaciones y prácticas pueden ser mejor representadas por verbos que indiquen el tipo de proceso que se trata de denotar, lo cual puede ayudar a llamar la atención acerca de los fenómenos sociales implicados en la creación y el uso de documentos digitales. Esto significaría que la simple idea de una taxonomía que únicamente refleja el orden del fenómeno, sería inapropiada al no enfatizar sus relaciones ni su naturaleza evolutiva. Es necesario darle mayor importancia al contexto para significar al documento digital.

Las reglas para construir una taxonomía de los términos que componen el lenguaje de la bibliotecología y disciplinas afines pueden obedecer a los siguientes principios:

6 Cfr. Gorbea, Salvador y Setien "De la bibliotecología al sistema de conocimientos científicos bibliotecológico-informativo” InvetigacónBiblicteclóġaardhivommáa, biblicteedoǵaeinformacón 8(16) pp. 26-27 ene-jun 1994 - en donde se sistematiza una alternativa para representar la jerarquía de los términos que representan actividades y conceptos. 
* Es necesario diseñar un esquema de clasificación que organice los conceptos disponibles por medio de categorías; es decir, que nos sirvan para incluir grupos de conceptos bajo un solo nombre.

* Las categorías que se diseñen deben arreglarse conforme a un orden primario preferido. Este orden preferido debe reflejar las relaciones significativas entre los términos que se utilizan para describir contenidos temáticos o la subordinación de un concepto a otro. Se entiende la jerarquía como una forma de ordenar por rangos de algún tipo, en donde el tipo superior incluya a los que se encuentren bajo él en la escala, la cual únicamente pretendería colocar los conceptos de clase en un orden lógico. Lo que se coloca en rangos distintos son las entidades y los niveles de organización o de significación.

La descripción de contenidos temáticos implica cuestiones terminológicas, pero además da lugar a instrumentos tales como los índices, los resúmenes y los sistemas de clasificación. Por su parte los índices y sistemas de clasificación implican un análisis temático, ${ }^{7}$ que requiere la comprensión del tema expuesto en el texto. Esta comprensión sería factible conociendo de los términos utilizados y sus correspondientes significados, por lo que un buen principio para la elaboración de índices sería la publicación de diccionarios terminológicos que regularmente se revisaran y actualizaran.

En el caso de la clasificación hay que considerar el aspecto semántico de los términos, puesto que para simbolizar los contenidos de manera tal que no existan ambigüedades hace falta que dicha simbolización se configure con base en contenidos o significados.

Por lo hasta aquí señalado puede verse cómo los aspectos morfológico, semántico y pragmático guardan estrecha relación al momento de realizar un análisis terminológico, e influyen de manera notable en las tareas propias de la bibliotecología.

Generalmente existe una tradición que sostiene el uso de estructuras temáticas que correspondan a sistemas científicos o filosóficos de la organización del conocimiento, sin embargo no siempre este tipo de sistemas son los mejores para determinar la organización temática.

\section{LA CLASIFICACIÓN DE LOS TÉRMINOS}

En principio para analizar el lenguaje de una disciplina se requiere determinar su copusdetéminos pero también son indispensables los conceptos que sustentan el diseño de un sistema clasificador para organizar este corpus y que permitan mostrar en forma sistemática el resultado del análisis. La organización de las relaciones entre los significados de los términos que constituyen el corpus del lenguaje de una disciplina debe servir para expresar los contenidos temáticos de ésta por medio de categorías

7 Examen de los documentos para la determinación de temas, que requiere entre otras cosas, la búsqueda de términos adecuados. 
que hagan explícitas las relaciones que existen entre estos contenidos. Mostrar las relaciones que existen entre los diversos conceptos de una disciplina facilita la obtención de una visión integrada acerca de la dinámica del lenguaje, y por ende del movimiento cultural que éste representa.

Plantearse la clasificación de los términos de que se compone el lenguaje de una disciplina como una actividad cognoscitiva, inherente al análisis del lenguaje y sus significados, permite hacer explícitas las relaciones entre los significados de los términos de los que se compone un lenguaje especializado. Al relacionar el significado de los términos por medio de un sistema clasificador se producen conocimientos acerca de la dinámica del lenguaje, con lo cual se obtiene una primera aproximación a los distintos cambios culturales, que representan la evolución de un lenguaje específico. Este es así porque el sistema clasificador permite mostrar los diversos niveles de organización jerárquica o la evolución cronológica de los significados, y la forma de representarlos por medio de uno o varios términos.

La formulación de relaciones entre los términos constitutivos del lenguaje especializado, implica un proceso de interpretación dirigido, por una parte, a agruparlos en torno a categorías y, por la otra, a dotar estas categorías de significados apropiados para crear un orden lógico capaz de expresar la diversidad de relaciones existentes entre los significados integrantes del cuerpo de conocimientos de una o varias disciplinas. Este proceso interpretativo genera conocimientos acerca de la composición de un lenguaje especializado, puesto que busca darle coherencia a la diversidad de términos y significados a la vez que explicar los vínculos que mantienen entre sí.

Los significados de los términos de un lenguaje especializado son representativos de un contexto cultural, es decir, se producen como resultado de una serie de actividades encaminadas a cumplir con determinados objetivos relacionados con la creación del conocimiento o con la intención de denotar un determinado tipo de evento. Aisladamente cada término expresa determinados significados, pero al reunirlo con otros se pueden obtener diversos significados. En la acción misma de clasificar los términos se encuentra una intencionalidad que dota a los términos de significado; así por ejemplo el uso de relaciones lógicas sustentadas en una cronología facilitaría la identificación del significado de determinados términos en etapas diferenciadas.

La clasificación de los términos de un leguaje especializado tiene como finalidad definir un universo que en primera instancia permita determinar si un término en particular puede, o no, incluirse como parte del universo de términos propios del lenguaje de una disciplina, pero además crea un espacio en donde se establecen relaciones lógicas entre los elementos pertenecientes a ese universo; relaciones que dotan de significado a cada uno de estos elementos y que sirven de base para organizarlos. Esta forma de clasificar resuelve los problemas específicos del lenguaje utilizado en la literatura hispanoamericana sobre bibliotecología, ciencias de la información y documentación, porque frente a conceptos ambiguos o contradictorios entre sí es factible establecer categorías de relación que integren los significados particulares en una totalidad (el esquema clasificador). Con lo cual si bien no se reduce la incertidumbre en el 
uso de los términos sí se posibilita la creación de instrumentos para comprender a qué obedece la diversidad de significados y de contenidos en el ámbito de las disciplinas antes mencionadas.

Si al ordenar la diversidad de conceptos inherentes al ámbito de la bibliotecología, ciencias de la información y documentación, consideramos a cada uno de ellos como un objeto independiente, pero al mismo tiempo estimamos que pertenece a un grupo de conceptos, se hace necesario no sólo interpretar, relacionar y jerarquizar situación que condiciona los significados de los términos considerados de forma aislada sino el contar con categorías que permitan identificar el tipo de relación que se establece. De esta manera la independencia de conceptos de los diferentes autores, creados conforme a factores ideológicos y culturales propios del entorno y creencias del autor, a la vez que se integran se diferencian, en razón del significado de las categorías utilizadas para distinguir las relaciones que se establezcan entre los significados de los términos. Los métodos para determinar la pertenencia de un término a una categoría que nombra una relación de significados entre términos, deben sustentarse en un proceso de inferencia que compruebe la validez de las condiciones para establecer cualquier tipo de relación entre los significados de diversos términos.

El manejo de las categorías de relación entre los significados que representan los términos, debe permitir distinguir los diversos significados atribuibles a un término, ya sea porque el contenido temático que exprese varíe conforme a la intención de delimitar un concepto, o bien se utilice para aseverar una apreciación distinta del objeto o fenómeno al que se alude. Un mismo término puede tener distintos significados dependiendo del contexto en que se utilice, así por ejemplo el término biblioteca, puede aludir a la función que ésta desempeña como institución social prestadora de servicios, o como una categoría que expresa un concepto acerca de las formas que tiene el ser humano para preservar y difundir el conocimiento.

La formalización de categorías para nombrar las relaciones entre los conceptos que integran el cuerpo de conocimientos del área que abarcan la bibliotecología y disciplinas afines es compleja por la diversidad de conceptos atribuibles a un mismo término, por ejemplo biblideca, doumeto, libro, dasificar, información en tre otros, que a veces se manifiestan en forma de ambigüedades en el momento en que un autor argumenta para justificar un fenómeno que trata de explicar. Esta situación provoca confusiones e incertidumbre porque existen términos vagamente definidos. La diversidad de conceptos atribuibles a un mismo término, es en ocasiones producto de situaciones culturales específicas, como puede ser la adopción indiscriminada de tecnologías para realizar actividades que son objeto de estudio de estas disciplinas. Adopción que se realiza en forma tan rápida que el conocimiento generado por la actividad científica de estas disciplinas se ve rebasado por el conimietoemṕnicoproducido por las soluciones adoptadas para solucionar los diversos problemas que plantea la prestación de servicios de todo tipo relacionados con la preservación y difusión de conocimientos, pero también deriva de posturas ideológicas que no se hacen explícitas so pretexto de mantener una actitud científica. 
La interpretación de las relaciones entre los significados de los términos de un lenguaje especializado se realiza a través de los objetos que tal lenguaje simboliza, donde las definiciones nominales se definen por símbolos, entendiendo por definición en el sistema lógico la asignación de un nombre breve a un complejo extenso de ideas.

$\mathrm{Si}$ entendemos por sistema un conjunto de elementos que guardan entre sí relaciones previamente definidas, nos encontramos que para clasificar los componentes de un lenguaje especializado, un sistema está conformado por elementos, como los términos y las relaciones lógicas, que muestren semejanzas y distinciones entre los significados que representan por medio de procesos de implicación e inferencia. El concepto implicar representa la idea de encontrar propiedades ya sea de igualdad o diferencia entre los elementos de una relación. La implicación presupone un conocimiento previo de los objetos por medio de la simple noción relativa de los elementos que la constituyen, y no incluye juicios de verdad o falsedad acerca de los elementos. $\mathrm{Al}$ efectuarse una relación entre los elementos que constituyen un objeto, la relación establece la verdad o falsedad de la propia relación.

Las propiedades lógicas de los elementos que constituyen los objetos, determinan la generalidad o particularidad de la relación sin que exista ningún juicio de valor. Un juicio de valor que se realizara respecto de los elementos sería un error, ya que se supone que se obtienen por medio de la intuición; es decir, mediante el recurso a la aprehensión inmediata de entidades perceptibles y de sus relaciones. De darse lo contrario, se cerraría la posibilidad de las relaciones lógicas posibles y se mantendrían principios absolutamente necesarios y no lógicos, y por tanto las propiedades no serían de relación y además no se podrían demostrar. Así por ejemplo, frente a la afirmación:

la biblicteea(elemento) tiene usuarios (propiedad), no permite establecer una deducción porque se carece de las posibilidades de la inferencia, que presenta la forma "si...ettones"

En este caso es mejor suponer:

si "la biblioteca tiene usuarios", entonces "la biblioteca tiene servicios".

Esta segunda demostración, al hacerse sucesiva, encadena las propiedades de los elementos; y cada conclusión se convierte en una premisa, que a su vez permite generar una nueva conclusión, que a su vez genera una nueva premisa, etcétera. Este encadenamiento origina una sucesión infinita que se interrumpe cuando se establece la relación necesaria conforme a un significado determinado.

El fundamento lógico de un sistema de clasificación se encuentra en la sistematización y la composición de un número infinito de relaciones entre los elementos que lo integran. Estas relaciones se pueden establecer entre un conjunto innumerable de términos pertenecientes a un lenguaje especializado, pero como el establecimiento 
de relaciones es susceptible de infinitas consideraciones no existe limitación alguna para el establecimiento de nuevos puntos de vista.

Un sistema de clasificación contiene conceptos que son considerados como indefinidos e inteligibles. Estos conceptos son llamados conceptos primitivos; la simplicidad lógica es también una noción relativa (una relación). Y para pasar de esas verdades absolutas a consecuencias, haciendo cadenas relacionales de razonamiento, hay que basarse en las propiedades lógicas de las relaciones, que son la base de la deducción. Para integrar un sistema clasificador los conceptos primitivos se obtienen por medio de la relación inmediata entre un objeto y otro, sin importar que éste sea de la misma especie o diferente. Los conceptos primitivos se manifiestan cuando ha sido explicado un fenómeno que de inmediato es identificado por un sujeto. El pensamiento del sujeto no puede unificar ni enlazar de algún modo, más que aquellos conceptos vinculados entre sí que existan previamente en el concepto primitivo, bien sea en su propia formulación o en los procesos del universo que representan respectivamente, o porque el sujeto tiene un orden previo que le permite jerarquizar los fenómenos implicados en el concepto.

\section{DE LAS RELACIONES EN UN SISTEMA DE CLASIFICACIÓN DEL LEN-} GUAJE

Un sistema de clasificación de un lenguaje especializado establece relaciones entre los términos que constituyen el lenguaje, el cual podemos concebir como una multiplicidad de términos que al vincularse recíprocamente en razón de sus significados obtienen como resultado un todo. Las relaciones entre los términos de un lenguaje especializado originan un orden unitario al relacionar dos términos que pertenecen a un mismo tema, o al establecer relaciones que refieren a temas distintos de un término a otro.

El propósito más general, a la vez que el más elemental y el más elevado de un sistema clasificador de un lenguaje especializado, consiste en delimitar un universo del lenguaje en su totalidad. Al término el sistema lo mantiene como una unidad para vincularlo con otros términos y formar un conjunto de términos pertenecientes a un tema, con lo cual adquiere un conocimiento general que se hace explícito al dividir el dominio completo de un área del conocimiento en campos separados, aun cuando no aislados, para formar en particular grupos especiales de relaciones de acuerdo con las propiedades de cada término. La formación de campos separados en un área de conocimientos se alcanza cuando se llegan a considerar solamente algunas características específicas del conocimiento generado por una disciplina, lo cual se logra haciendo una abstracción transitoria de aquellos aspectos que se incluyen en el punto de vista establecido en la formación de los campos contemplados para la organización del conocimiento. 
Si nos acercamos al cúmulo de términos que podrían constituir un corpus de términos de bibliotecología, lo primero que tendríamos que plantearnos es cómo establecer las relaciones entre estos términos. Estas relaciones se iniciarían mediante el proceso de implicación de un término con otro por medio de la simple percepción de los elementos que integran el significado de un término que conduce a la relación inmediata con los elementos de cada uno de los términos implicados. Si por ejemplo, tomamos el término: dasificacón dbametal

En primer lugar podríamos pensar en la posibilidad de una

1.- Teana delaClasificación deDoumentos que entre otras finalidades pueda determinar

2.-Méodbsparad DiseñodeSistemasdaClasificación , que a su vez sirvan como instrumento para el

\section{3.- DiseñodeSistemas deClasificación}

En este ejemplo el término clasificación documental establece un significado de la clasificación referida al ámbito documental, y se implica con otros tres términos compuestos cuyo significado está relacionado con la clasificación documental, aunque los significados de cada uno de los términos implicados sean de distinta naturaleza. En este caso no importa la cualidad de cada significado sino su calidad de poder ser referido a la clasificación documental, la relación inmediata por medio de este término tiene la finalidad de identificar a cada uno de los términos como unidades dependientes; es decir pertenecientes a un campo temático determinado, pero que son independientes porque mantienen sus propios significados. Las relaciones clasificadoras no cambian su significado particular, únicamente introducen la noción de pertenencia a un determinado conjunto temático.

Una relación de pertenencia entre los términos de un conjunto temático se refleja en el significado propio de cada término, pero también lo convierte en un miembro de un conjunto temático dotándolo de nuevos elementos de significación. A partir del significado de un término, en el entendido de que éste exista como tal, un sujeto puede explorar los elementos de que se compone su significado como fenómenos independientes, con lo cual origina una primera abstracción por medio de la cual se obtiene una noción del término objeto de análisis. Esta abstracción del significado del término permite concebirlo como un todo inseparable, susceptible de descomponerse en elementos que pueden vincularse entre sí o con los elementos temáticos de otro documento.

El corpus de términos de la bibliotecología podría relacionarse por medio de los diversos significados de cada uno de los términos representan qué es y cómo es la disciplina. Por ejemplo, podemos decir que el término análisisdoumental forma parte de los fenómenos propios del estudio de la bibliotecología porque la relación de implicación establecida por el conocimiento bibliotecológico nos permite saber que existen términos que hacen relación alusiva al análisis documental. Establecer relaciones entre el significado de varios términos implicados entre sí requiere identificar el concepto que expresan las palabras que enlazan los significados de los conceptos 
implicados y que dan lugar a un tipo de relación, y por tanto debe tener un nombre que distinga esa relación de otras. Así por ejemplo, examinemos lo siguiente:

Los conceptos de mótodos pararesumir, reseñar, yrealizar estados di arte, implican de suyo el de análisis dbamental, de manera que podemos considerar que existe una relación entre los significados de estos términos.

Si consideramos a los términos antes mencionados como un conjunto de términos (elementos de representación temática) de un lenguaje especializado, podemos establecer relaciones entre sus significados. Sin embargo, la formulación de relaciones entre los significados de los términos requiere precisar antes varios factores:

* Para asociar y contextualizar conceptos lo obvio es determinar con claridad el concepto del que se va a partir para hacer las asociaciones.

* Igualmente hay que especificar la manera en que se relacionan los significados entre sí, en este caso podríamos decir que los mútodbspara resumir, resenar, yrealizar etadosdd arte implican el análisis documental porque las definiciones de estos conceptos presuponen el análisis del documento como un concepto previo. Por ejemplo si consideramos el concepto resumir, podemos considerar que en él está implicado el concepto de análisis documental en tanto que resumir requiere descomponer en partes los contenidos de un documento para poder representarlo por medio de un resumen.

* También es necesario determinar la naturaleza de la relación que hay que establecer. Si construimos una relación que especifique una jerarquía de lo general a lo particular, o bien de lo particular a lo general, tendríamos que distinguir el término que mantiene el primer orden jerárquico de ser general, para después circunscribir los términos particulares. En cambio podríamos considerar que la relación no expresa una jerarquía sino meramente una afinidad semántica, en cuyo caso únicamente sería necesario expresar la relación.

Sería ineludible estipular el nombre de la relación; es decir, tenemos los conceptos y la forma en que se relacionan entre sí, pero sería necesario darle un nombre a esta relación. Este nombre es el que permite ubicar este conjunto de términos del que nos ocupamos como parte de un esquema clasificador. En este caso podríamos nombrar esa relación con el término de análisis documental, dado que su significado se encuentra implicado con el de los tres términos mencionados; es decir es el concepto de mayor grado de generalidad.

Si consideramos al término análisisdoumental como primer orden de ser general, entonces todos los demás términos son particulares del término análisisdbamental. En teoría todo término que sea general subordina a los otros términos y éstos serán particulares de ese término general. La representación de una jerarquía formal entre términos permite, cuando se relacionan, establecer varios y diversos significados de ellos, lo que posibilita la resignificación de términos de acuerdo con un significado preestablecido. 
En este caso tendríamos un conjunto de términos relacionados entre sí dado que el concepto de resumir, reseñar y hacer un estado del arte implican el análisis documental, y decidimos además nombrar a la relación con el nombre de análisis documental. No obstante esta primera relación podemos inscribirla como parte de un sistema clasificador por medio del establecimientos de nuevas relaciones entre las relaciones ya definidas, o bien podemos organizar las relaciones establecidas por medio de un orden alfabético que facilite su consulta. Tendríamos entonces las siguientes opciones:

* establecer relaciones entre una relación genérica y varias particulares

* jerarquizar las relaciones definidas

* organizarlas por orden alfabético

* recurrir a un mapa de relaciones que nos muestre las afinidades semánticas entre las relaciones definidas

Si consideramos la relación Análisis Documental como un relación genérica, entonces puede también relacionarse con otras relaciones de términos particulares, como se muestra a continuación:

\section{Análisis documental $\Rightarrow$ Teorías del Análisis Documental \\ $\Rightarrow$ Teorías de la Representación Documental}

En este ejemplo existe una relación de implicación de términos porque se tiene una noción relativa para indicar que las teorías del análisis documental y las teorías para la representación de documentos, pueden formar parte del análisis documental. El mismo tipo de implicación se presenta en el siguiente ejemplo:

\section{Análisis documental > Métodos para Resumir}

Sin embargo al relacionar el término análisis documental con términos particulares como: > teorías del análisis documental > teorías de la representación documental $>$ métodos para resumir, la implicación no establece una jerarquía sino relaciones entre términos.

$\mathrm{Si}$ optamos por jerarquizar las relaciones definidas es necesario establecer una jerarquía, que ordene las relaciones. Entonces el nombre de la relación que establecimos como análisis documental adquiere significación por la representación jerarquizada de las relaciones con otras relaciones cuyo significado guarda algún vínculo con el significado del término análisis documental, lo cual aumenta las posibilidades de que surjan relaciones lógicas entre los diversos elementos que constituyen un sistema.

Las relaciones lógicas de un sistema son relaciones de significación, es decir, la relación se construye en razón del significado que se desea obtener. Una relación derivada del significado de los mítodbsdeamálisisdboumetal establece un significado: la calidad de método para analizar. En este caso la relación busca vincular diversos tipos de métodos: para resumir, para evaluar, para reseñar, etcétera, los cuales tienen un significado propio, por ejemplo, el procedimiento que se sigue para resumir y enseñar a resumir. No obstante la relación los cualifica como parte del análisis documental. 
Al implicar los elementos que integran el análisis documental, por ejemplo, podemos decir que consta de métodos, teorías, etcétera, y que todos estos elementos conforman una estructura lógica. El análisis documental constaría de elementos tales como:

* Teorías de análisis documental

* Teorías de representación documental

* Análisis de contenido

* Métodos para conocer el estado del arte

* Análisis terminológico

* Métodos para resumir

* Métodos para reseñar

* Metría de los documentos

* Métodos para hacer análisis documental

* Calidad de la información

* Métodos para evaluar documentos

* Métodos para hacer selección documental

Mismos que constituyen una estructura lógica.

Al formar una estructura, los elementos que integran el análisis documental, pueden integrarse dentro de un sistema clasificador como lista de conceptos, y las relaciones lógicas de un sistema vinculan cada una de las listas completas de los conceptos con cada uno de los significados de términos en particular dotándolos de significado. Una lista de conceptos puede agrupar conceptos que se refieren a objetos o a fenómenos, y por medio de una relación podemos diferenciar cada uno de los tipos de conceptos y establecer relaciones de significación como las siguientes:

"análisis formal"

"análisis temático"

"análisis de contenido"

Una relación puede darse también entre dos grupos de conceptos distintos como, por ejemplo, buscar y reuperar, por medio de la cual podrían producirse nuevos significados para buscar y recuperar documentos e información, en tanto la relación puede atribuirle la pertenencia a determinado tema. Las relaciones de un sistema producen significados que permiten averiguar diversas posibilidades sobre el concepto de los elementos implicados en buscar y recuperar documentos e información, porque se pregunta por la relación y no por el concepto en sí. Así, una vez localizada la relación ésta nos conduce a uno o varios términos.

Buscar y recuperar documentos e información nos obliga a tener un mayor conocimiento sobre los posibles elementos que constituyen la organización documental, a partir de los cuales se establece la pertenencia de éstos a una determinada relación. Intervienen diversos factores para establecer relaciones en un sistema, si tomamos el término Estruduradelacommicaaónaentíficapodemos relacionarlo con la idea de buscar y recuperar documentos e información en tanto los modos de comunicación científica 
determinan los modos de difusión, circulación y preservación de documentos e información científica, elementos indispensables para buscar y recuperar ésta.

La Estructura dela commicacón doumetal tiene relación con buscar y recuperar y con la Estructuradelacammicadónaientífica, debido a que le atribuimos un significado común: son formas de comunicación. Sin embargo no podemos dejar de considerar las diferencias de significado que existen entre el término Commicaaóndoumental y el de Comuicadóncientífica ni que la relación se establece únicamente en razón de buscar y recuperar documentos e información, porque si tratamos de relacionar el término Commicadóndbamental con el de Commicadóndientífica, tenemos que considerar que los significados de comunicación científica no siempre arrojan una significación atribuible a la comunicación documental.

En el caso anterior las relaciones se establecen entre diferentes términos a los cuales se les atribuye un significado común que sirve como sustento la relación.

\section{SÍMBOLO, TÉRMINO, SIGNIFICADO}

Los términos forman parte del lenguaje y tienen, por tanto, su propia sintaxis gramatical, a partir de la cual se establecen sus significados y sus relaciones. La palabra, como una de las unidades más pequeñas del lenguaje, tiene un significado propio y conforme se relaciona con otras palabras adquiere un nuevo significado.

Las formas de relaciones gramaticales entre distintos términos obedecen a una construcción de oraciones; sin embargo en un sistema pueden construirse relaciones temáticas que obedezcan a una jerarquía previamente determinada. Es decir que las relaciones entre términos de un sistema presuponen la existencia de la forma en que van a darse esas relaciones, y de las cuales se habló anteriormente.

Las relaciones lógico-formales entre términos se sustentan en su capacidad de simbolizar. Si sucede que un símbolo no representa a ningún objeto en particular sino que tiene un grado de abstracción que nombra diversos objetos con características semejantes, entonces las relaciones lógicas entre términos se darían con base en los objetos que nombran y no únicamente por el significado semántico del término, por ejemplo: doumento

La interpretación de las relaciones entre los elementos se realiza a través de los objetos que simboliza; estas relaciones son indefinidas porque el símbolo solamente agrupa objetos diversos que tienen características semejantes, y estas características se obtienen con la definición de un término. En un sistema lógico entendemos por definición la asignación de un nombre breve a un complejo extenso de ideas. Tales definiciones son nominales, lo que se define es un símbolo.

Desde este punto de vista las definiciones son convenciones simbólicas. Pero siempre que surge el problema de la interpretación la selección de los conceptos que deben ser definidos se torna de la mayor importancia, porque implica la existencia 
previa de las relaciones lógico-formales, por medio de las cuales se van a dar las relaciones entre términos, puesto que esta selección determinará la naturaleza del sistema.

\section{REFERENCIAS BIBLIOGRÁFICAS POR ORDEN ALFABÉTICO DE AU- TOR}

García Marco, Francisco Javier.."Vannevar Bush, el hipertexto y el futuro del documento".- En: Tendanasdelainvestigaiónen docmentacoón- Editado por Jesús Tramullas. Zaragoza, España: Seminario TID, 1996.- 185-210

Lancaster, Frederick W. El contrd dd vocabulanioenla reuperacón de información- 2a. ed.- Valencia, España: Universitat de València, 1995. 286 p.

Lynch, Clifford. "La exploración de Internet". en: Investigacóny Cienia(Edición Española de Scientific American), mayo de 1997, p.38-43.

Ranganathan, R. S. Classified CatalogreCode With additional nules for Didionary CatalogueCode India: Sarada Ranganathan Endowment for Library Science, 1989. 644 p.

Setien, Emilio y Salvador Gorbea "De la bibliotecología al sistema de conocimientos científicos bibliotecológico-informativo" Investigacoón Biblideedógica ardivonmmá, biblictedoǵa e infomadón 8 (16) ene-jun 1994, 63 p.

Tomasini Bassols, Alejandro.- LenguajeyAnti-mtafísica: CaviladonesWinttgensteinianas- México: INBA/Interlínea, 1994.- 282 p. .- p. 31 y ss.

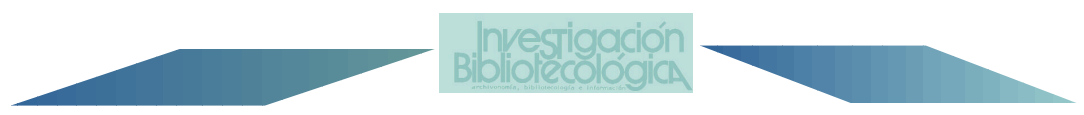

